\title{
Genome-wide analysis of expansin superfamily in wild Arachis discloses a stress-responsive expansin-like B gene
}

\author{
Larissa Arrais Guimaraes ${ }^{1}$ Ana Paula Zotta Mota ${ }^{1,2} \cdot$ Ana Claudia Guerra Araujo ${ }^{1}$. \\ Lucio Flavio de Alencar Figueiredo $^{3}$ - Bruna Medeiros Pereira ${ }^{1,3} \cdot$ Mario Alfredo de Passos Saraiva $^{1}$. \\ Raquel Bispo Silva ${ }^{1,3} \cdot$ Etienne G. J. Danchin $^{4} \cdot$ Patricia Messenberg Guimaraes $^{1} \cdot$ Ana Cristina Miranda Brasileiro ${ }^{1}$
}

Received: 7 October 2016 / Accepted: 13 February 2017 / Published online: 27 February 2017

(C) The Author(s) 2017. This article is published with open access at Springerlink.com

\begin{abstract}
Expansins are plant cell wall-loosening proteins involved in adaptive responses to environmental stimuli and various developmental processes. The first genome-wide analysis of the expansin superfamily in the Arachis genus identified 40 members in A. duranensis and 44 in A. ipaënsis, the wild progenitors of cultivated peanut (A. hypogaea). These expansins were further characterized regarding their subfamily classification, distribution along the genomes, duplication events, molecular structure, and phylogeny. A RNA-seq expression analysis in different Arachis species showed that the majority of these expansins are modulated in response to diverse stresses such as water deficit, rootknot nematode (RKN) infection, and UV exposure, with an expansin-like B gene (AraEXLB8) displaying a highly distinct stress-responsive expression profile. Further analysis of the AraEXLB8 coding sequences showed high conservation across the Arachis genotypes, with eight haplotypes
\end{abstract}

Accession Numbers The AraEXLB 8 coding sequences from 13 Arachis genotypes have been deposited at NCBI GenBank under the accession KX588113 to KX588125.

Electronic supplementary material The online version of this article (doi:10.1007/s11103-017-0594-8) contains supplementary material, which is available to authorized users.

Ana Cristina Miranda Brasileiro

ana.brasileiro@embrapa.br

1 Embrapa Recursos Genéticos e Biotecnologia, Parque Estação Biológica, Final W5 Norte, Brasília, DF CP 02372, Brazil

2 Universidade do Rio Grande do Sul, Porto Alegre, RS, Brazil

3 Universidade de Brasília, Campus Darcy Ribeiro, Brasília, DF, Brazil

4 Institut Sophia Agrobiotech, INRA, University of Nice Sophia Antipolis, CNRS, 06900 Sophia Antipolis, France identified. The modulation of AraEXLB8 expression in response to the aforementioned stresses was confirmed by qRT-PCR analysis in distinct Arachis genotypes, whilst in situ hybridization revealed transcripts in different root tissues according to the stress imposed. The overexpression of AraEXLB8 in soybean (Glycine max) composite plants remarkably decreased the number of galls in transformed hairy roots inoculated with RKN. This study improves the current understanding of the molecular evolution, divergence, and gene expression of expansins in Arachis, and provides molecular and functional insights into the role of expansin-like B, the less-studied plant expansin subfamily.

Keywords Drought $\cdot$ Meloidogyne . Ultraviolet (UV) · Composite plant $\cdot$ Glycine max

\section{Introduction}

Expansins are cell wall loosening proteins involved in extension and relaxation of cells in growing tissues through a non-enzymatic activity (McQueen-Mason et al. 1992). Plant expansins are also implicated in responses to many abiotic and biotic stresses, such as drought; salinity; cold; heat; oxidative stress; herbivore attack and phytopathogen infection (Marowa et al. 2016), indicating that these proteins constitute a common component in the response of plants to stress. The role of expansins in stress responses has been reinforced by studies showing that the ectopic overexpression in transgenic plants of some expansincoding genes leads, in general, to enhanced overall plant growth and tolerance to abiotic stress (Cosgrove 2015; Marowa et al. 2016; Sasidharan et al. 2011). In contrast, the reduction of expansin transcripts, which inhibits cell expansion, leads to an increase in resistance to plant diseases 
possibly due to a more efficient cell wall physical protection (Ding et al. 2008; Gal et al. 2006). Expansins are also involved in responses to oxidative stress (Han et al. 2015) derived from enhanced production and accumulation of Reactive Oxygen Species (ROS) (Baxter et al. 2014). As a secondary stress, oxidative damage is generally ubiquitous in almost all types of stresses, including ultraviolet (UV) exposure, thus supporting the idea that expansins are common and crucial players in the response to multiple and simultaneous stresses. Nevertheless, the non-enzymatic mechanisms by which expansins influence the ability of plants to withstand associated stresses remain uncertain.

The expansin superfamily is ubiquitous in the plant kingdom and classified into four subfamilies: $\alpha$-expansin (EXPA), $\beta$-expansin (EXPB), expansin-like A (EXLA) and expansin-like B (EXLB) (Kende et al. 2004). It has been assumed that expansin genes belonging to the same phylogenetic group share almost similar functions and effects regarding plant development and growth (Marowa et al. 2016). To date, research efforts have been concentrated on elucidating the mode of action and the biological function of expansins belonging to the two largest subfamilies, EXPA and EXPB, and, to a lesser extent EXLA, whilst little attention was given to the EXLB members (Cosgrove 2016; Marowa et al. 2016).

Recently, genome-wide investigations of expansins in species belonging to Fabaceae family, such as Glycine max (soybean), Phaseolus vulgaris (common bean) and Medicago truncatula (Cosgrove 2015; Liu et al. 2015; Zhu et al. 2014) have brought new insights into the molecular and evolutionary history of expansins in legumes, as well as their functions and regulatory mechanisms. In G. $\max$, the expansin superfamily has expanded predominantly by segmental duplication and presents a broad functional divergence among subfamilies (Zhu et al. 2014). Likewise, studies in $M$. truncatula revealed that both segmental and tandem duplications contributed to the evolution and diversification of expansins and that genes involved in same processes are closely located on chromosomes (Liu et al. 2015). In this regard, the recently available genome sequences of Arachis duranensis and A. ipaënsis, the wild progenitors of cultivated peanut (A. hypogaea) (Bertioli et al. 2016; Chen et al. 2016), represents a great opportunity to advance the knowledge on plant expansins in Arachis and other legumes.

Wild Arachis species have been exploited in the last years as sources of alleles to enhance environmental adaptability and to disclose candidate genes, genetic markers, and genomic sequences for peanut breeding improvement (Brasileiro et al. 2014; Janila et al. 2016). In this context, previous transcriptome analysis revealed several differentially expressed genes responsive to drought, including expansin genes, in A. duranensis and A. magna (Brasileiro et al. 2015; Guimaraes et al. 2012). Moreover, A. stenosperma transcriptome survey identified, amongst other candidates, expansin genes putatively involved in resistance to the root-knot nematode (RKN) Meloidogyne arenaria (Guimaraes et al. 2015). Either through harboring improved performance under water-limited conditions ( $A$. duranensis and A. magna) or higher resistance to several pathogens (A. stenosperma), these wild species proved to be valuable for stress-related gene discovery.

The potential of expansin-coding genes to enhance stress tolerance, associated with the limited knowledge of their roles in plant responses to environmental cues, highlights the importance of characterizing the expansin superfamily and their functional mechanisms, in order to enable their use for plant improvement. The present study reports the first genome-wide identification and analysis of the expansin superfamily in the genus Arachis and the molecular and functional characterization of a novel stress-responsive expansin-like B gene (AraEXLB8), a member of the lessstudied expansin subfamily.

\section{Results}

\section{Genome-wide identification and analysis of Arachis expansins}

Overall, 40 and 44 putative expansins were identified in the genomes of A. duranensis and A. ipaënsis, respectively, with most of them ranging between 250 and 275 amino acids (AAs) in size and harboring signal peptides for secretion (Supplementary Tables 1 and 2), as expected from canonical plant expansins (Sampedro and Cosgrove 2005). Using a phylogenetic analysis, most of the Arachis expansin genes could be assigned to the four subfamilies proposed by Kende et al. (2004) with high confidence values (Fig. 1a; Supplementary Figs. 1, 2, 3). The phylogeny showed that EXPA, EXPB and EXL (A and B) subfamilies formed highly supported monophyletic groups. However, within the EXL group, EXLB proteins did not form a highly supported monophyletic group but were interspersed by the highly supported EXLA monophyletic group, although this separation of the EXLBs in two groups received only a moderate bootstrap support value of 47 (Fig. 1a). A further phylogenetic analysis, including only EXLAs and EXLBs, confirmed either the separation of EXLB in two subgroups (Supplementary Figs. 4a, 5) or low support for the monophyly of EXLBs (Supplementary Fig. 4b). Among A. duranensis expansins, the EXPA subfamily constituted the largest clade with 25 members, followed by eight EXLB, six EXPB and one EXLA member, whilst for A. ipaënsis, expansins were classified as 


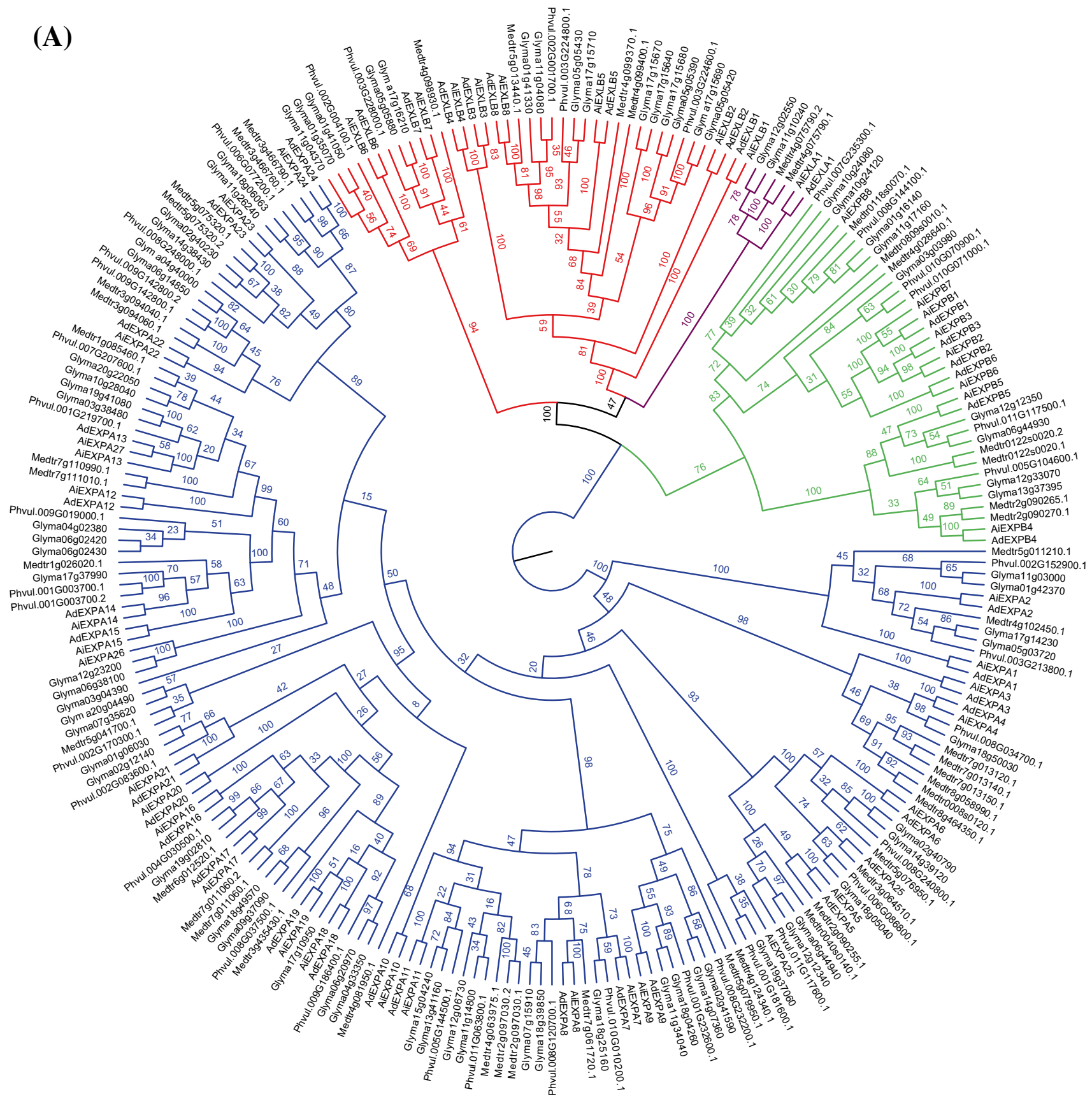

Fig. 1 Phylogenetic analysis and expansin distribution in Arachis duranensis and Arachis ipaënsis. a Phylogenetic analysis of the expansin superfamily in A. duranensis (Ad); A. ipaënsis (Ai); Glycine max (Glyma); Phaseolus. vulgaris (Phvul) and Medicago truncatula

27 EXPA, eight EXLB, eight EXPB, and a single EXLA (Fig. 1a; Supplementary Tables 1, 2, 3).

Expansins were unevenly distributed on all the chromosomes of both Arachis species (Fig. 1b). Whilst the EXPA members (blue) were distributed on each chromosome of both species, EXLB (red) and EXPB (green) tend to be grouped on chromosomes 1 and 2, respectively, and appear
(Medtr). b Distribution of expansin genes in the ten chromosomes of each A. duranensis (light gray; A01-A10) and A. ipaënsis (dark gray; B01-B10). Synteny between the two genomes is represented by lines. EXPA (blue), EXPB (green), EXLA (purple) and EXLB (red)

to be mutually exclusive in their locations in each species. Similar uneven distribution of the EXLB members, clustered in one or two chromosomes, was previously observed in Malus x Domestica (Zhang et al. 2014b) and G. max (Zhu et al. 2014).

Whole genome level analysis showed that the majority of the predicted protein-coding genes for A. duranensis 


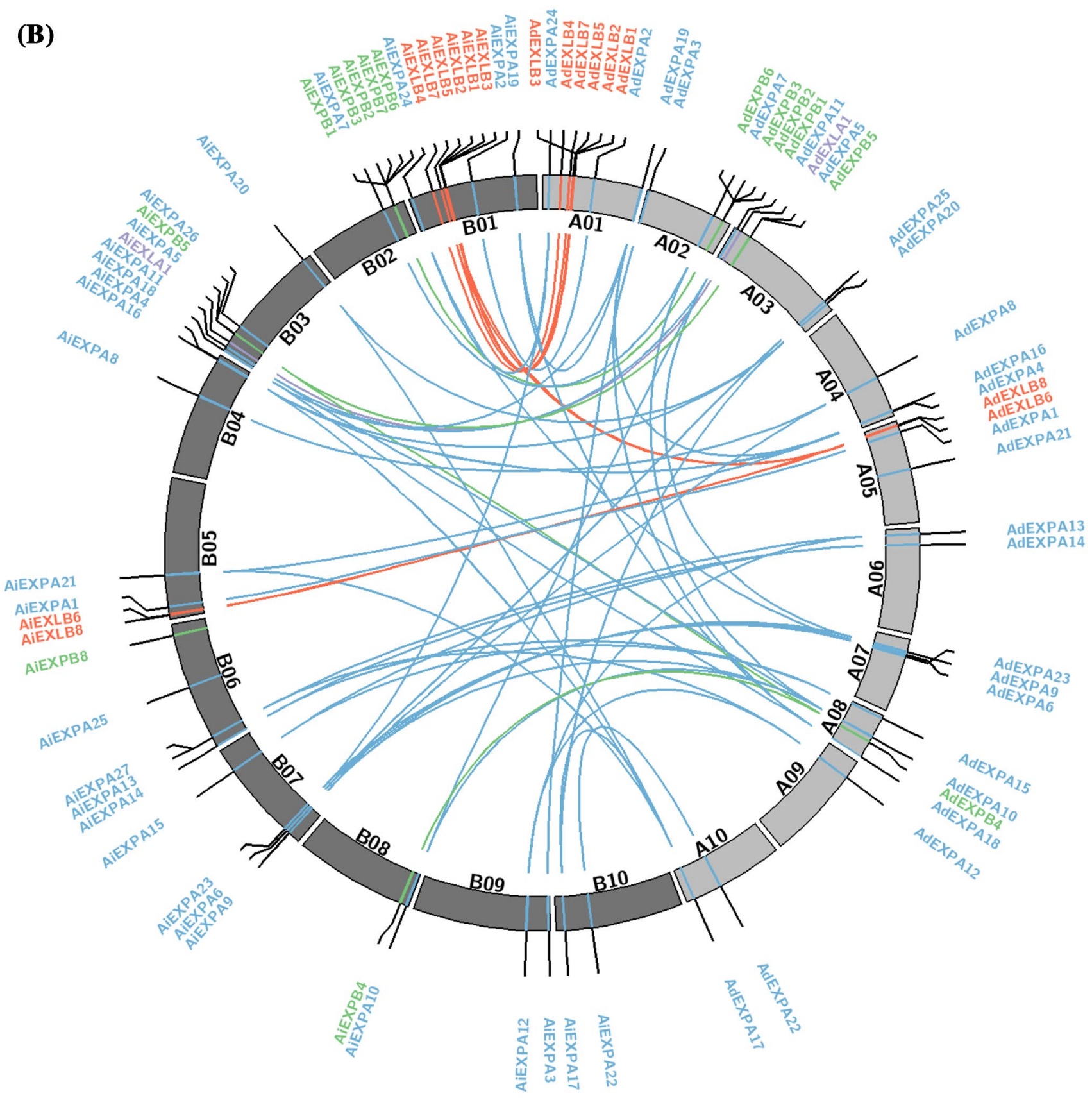

Fig. 1 (continued)

(87.2\%) and A. ipaënsis (88.6\%) were duplicated. In both genomes, these duplicated copies resulted mostly from dispersed gene duplication $(>68 \%)$ all around the genomes, whilst less than $17 \%$ resulted from whole genome duplication (WGD) (Supplementary Table 4). In contrast, expansion of the expansin superfamily genes mainly resulted $(>50 \%)$ from WGD/segmental duplication events in both species rather than from dispersed duplications (Supplementary Table 7). Within the expansin subfamilies (Supplementary Table 5), the most common type of duplication for EXPA and EXLB was WGD/segmental duplication, whilst for EXPB, tandem duplications represented the majority. Further analysis also showed that the expansion of the expansin subfamilies in Arachis was comparable to that observed in Arabidopsis thaliana and G. max (Supplementary Table 5).

To predict the selective pressure between Arachis expansins duplicated genes, the ratio of $K a$ (rate of non-synonymous mutations) $/ K s$ (rate of synonymous mutations) was calculated and revealed that only the 
AiEXLB5/AiEXLB8 genes pair from A. ipaënsis appeared to have undergone positive selection $(K a / K s$ ratio $>1)$.

Phylogenetic analysis indicated that most duplication events of the expansin genes preceded A. duranensis and A. ipaënsis divergence (Fig. 1a) and might have occurred in a common ancestor, most probably since the divergence of the Dalbergioid clade (Bertioli et al. 2011). Although large inversions of both arms have been previously observed in chromosome 1 of Arachis species (Bertioli et al. 2016), most of the conserved syntenic blocks of duplicated expansins are located on chromosomes 1, 2 and 3 (Fig. 1b). Moreover, the high density of expansin genes in A. duranensis chromosome 8 is in accordance with the gene-rich characteristic of this abnormally small 'A chromosome' that contains low repetitive content and many genes (Bertioli et al. 2016).

\section{Arachis expansin gene and protein structures}

Arachis duranensis and A. ipaënsis shared conserved expansin gene structures (Fig. 2) and showed diverse organizations comprising of two to six exons. Members of the same phylogenetically-determined subfamily were characterized by a similar intron/exon organization; in particular, those of the EXPB subfamily, in which all members consisted of four exons, except AiEXPB8. The majority of the EXPA members (82\%) had three exons, while EXLB genes presented the largest number of exons, ranging from four to six, similar to Malus x Domestica and G. max (Zhang et al. 2014b; Zhu et al. 2014). The EXLA members had the same gene structure (4-exon/3-intron) in both Arachis species which differs from the usual 5-exon/4-intron EXLA structure of other plants (Ding et al. 2016; Krishnamurthy et al. 2014; Sampedro et al. 2005).

Predicted expansin protein structures (Fig. 2) showed a highly conserved pattern of DPBB and CBM63 Interpro domains (Cosgrove 2015) (Supplementary Figs. 2, 3), preceded by a signal peptide with 21 to $36 \mathrm{AA}$ in most N-terminus. Also, the highly conserved intron/exon structure of Arachis expansins within each subfamily supports the classification proposed here, as well as the evidence of the close evolutionary relationship among them. Two EXPA members (AdEXPA3 and AdEXPA13) contained unusual additional copies of DPBB or CBM63 domains in their predicted AA sequences. Since these atypical structures could be the result of gene prediction errors, they were not considered for further characterization.

\section{RNA-seq expression profiling of expansin genes under biotic and abiotic stresses}

In order to gain broader understanding of the Arachis expansin superfamily gene regulation in response to stress, our previous transcriptome data of $A$. duranensis and A. stenosperma plants submitted to abiotic (water deficit and UV exposure) and biotic (RKN $M$. arenaria inoculation) stresses were here exploited. The expression profile (Fig. 3) indicated that all the Arachis expansin genes were modulated in response to at least one of the imposed stresses, with distinct expression behaviors depending on the species and stress. The hierarchical clustering analysis classified the expansin genes into five clusters (A-E) according to similarities in their transcript abundance patterns (Fig. 3). Clusters $\mathrm{A}$ and $\mathrm{C}$ were the most distinct, as each harbored only one representative, $A d E X L B 8$ and AdEXPB6, respectively, as a result of their unique expression profiles. Clusters $\mathrm{B}$ and $\mathrm{D}$ contained a similar amount of genes (14 and 13, respectively), encompassing representatives of three subfamilies (EXPA; EXPB and EXLB), whereas cluster E, with nine genes, included a single member of EXLA subfamily (AdEXLA1) but no representatives of the EXPB subfamily. Genes forming cluster $\mathrm{D}$ showed slight variations on their expression levels in response to the variable stresses applied. Differently, higher levels of gene expression and contrasting expression behaviors of A. duranensis expansin genes and A. stenosperma orthologs were observed between clusters B and E (Fig. 3). In cluster E, the majority of expansins were upregulated in A. stenosperma orthologs upon $M$. arenaria inoculation in roots (SN3, SN6 and SN9) or UV exposure in leaves (SUV), whereas a general downregulation was observed in A. duranensis (DN3, DN6 and DN9; Fig. 3). Interestingly, in cluster B, the opposite expression behavior occurred in response to M. arenaria infection (Fig. 3), in particular for AdEXLB1, $A d E X L B 3$ and $A d E X P B 3$ genes, which showed a strong upregulation in $A$. duranensis. Regarding the drought imposition treatment, however, the expression profile displayed by expansins belonging to clusters $\mathrm{B}, \mathrm{C}$ and $\mathrm{E}$ (Fig. 3) were quite similar between A. duranensis (DDro) and A. stenosperma orthologs (SDro). These results suggest that Arachis expansin genes may exhibit both functional differentiation in the two species and overlapping responses between abiotic and biotic stresses.

An expansin-like B gene (EXLB8) has drawn attention as it represented an outlier among the Arachis expansin genes, with a highly specific expression in response to each stress (Fig. 3). Moreover, our previous studies suggested the involvement of EXLB8 in RKN-resistance in A. stenosperma (Guimaraes et al. 2015), as well as in drought tolerance in A. duranensis and A. magna (Brasileiro et al. 2015). EXLB8 gene from wild Arachis species (hereafter named AraEXLB8) was therefore selected for a broader characterization at molecular and functional levels, as the most promising candidate, among the Arachis expansin genes, to be further exploited for plant genetic engineering. 

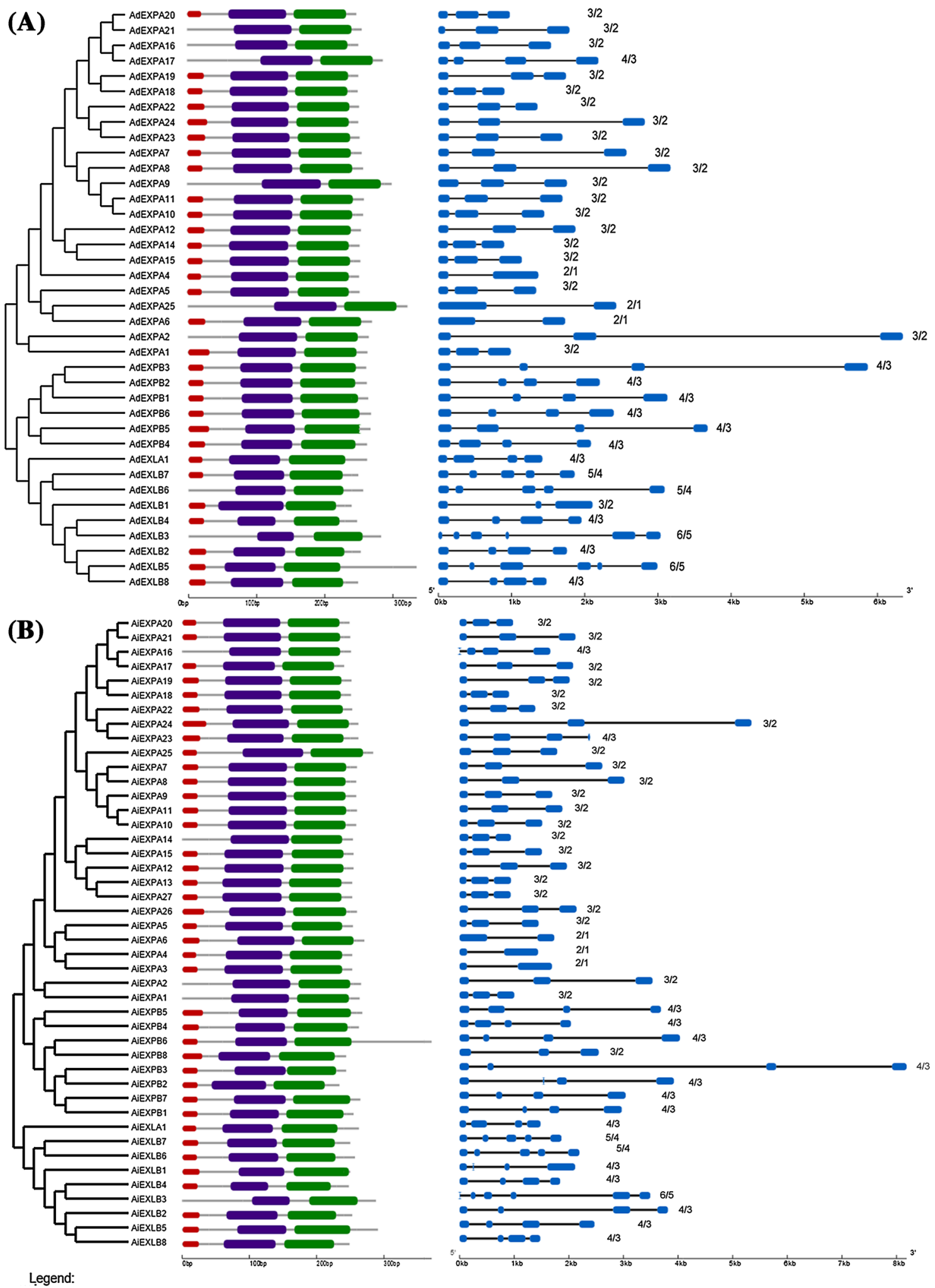

CBM63 DPBB $\square$ signal Exon - Intron

Fig. 2 Protein and exon/intron gene structure of expansins in a Arachis duranensis and $\mathbf{b}$ Arachis ipaënsis. Protein structure diagram (left): DPBB and CBM63 domains are represented by purple and green boxes, respectively, and peptide signals by red boxes. Gene structure diagram (right): exons are represented by blue boxes and introns by linking single lines. The number of introns and exons, respectively, is after each diagram 

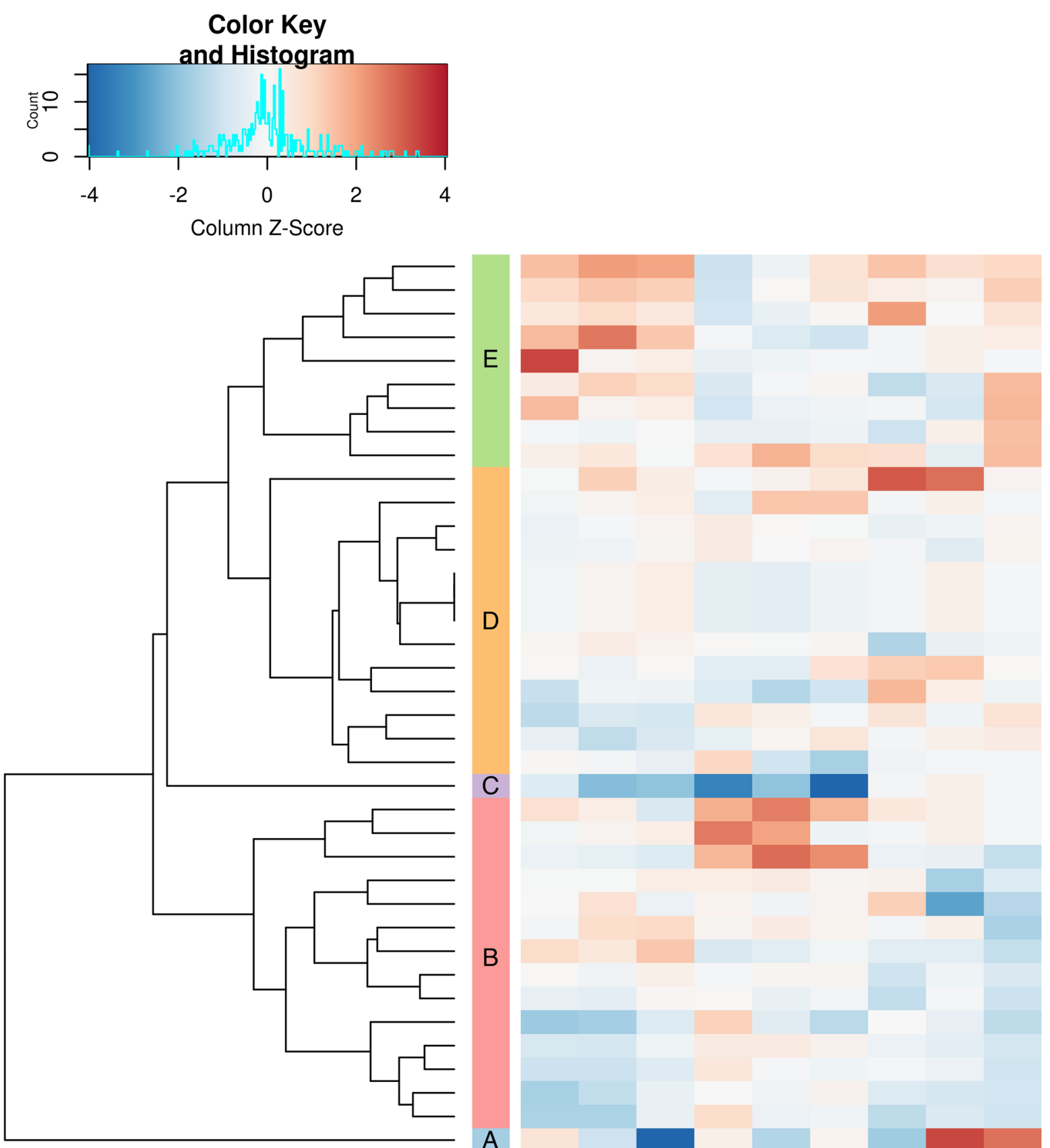

AdEXLA1

AdEXPA14

AdEXPA23

AdEXLB2

AdEXPA2

AdEXPA12

AdEXPA19

AdEXPA18

AdEXLB4

AdEXPA24

AdEXPA15

AdEXPA10

AdEXPA9

AdEXPA4

AdEXPB1

AdEXPB2

AdEXPA25

AdEXPA17

AdEXLB6

AdEXPA20

AdEXPA8

AdEXLB5

AdEXPB6

AdEXLB3

AdEXPB3

AdEXLB1

AdEXPA5

AdEXLB7

AdEXPA16

AdEXPA1

AdEXPA6

AdEXPA7

AdEXPA22

AdEXPB4

AdEXPA11

AdEXPB5

AdEXPA21

A

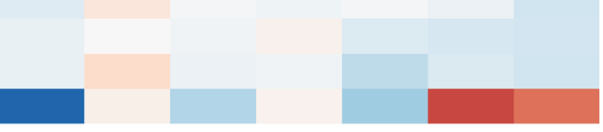

AdEXLB8

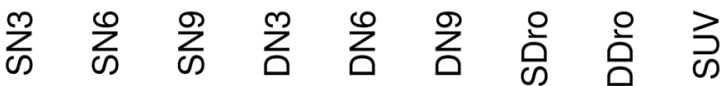

Fig. 3 Heatmap of expansin transcripts of Arachis duranensis genes and Arachis stenosperma orthologs. Expression patterns (log2-based values) in roots of A. duranensis (D) expansin genes and A. steno-

\section{Characterization and phylogenetic analysis of AraEXLB8 gene}

The 13 AraEXLB8 coding sequences here identified (NCBI accession numbers KX588113 to KX588125), together with three additional available sequences, were used for sperma orthologs (S) at 3 (N3), 6 (N6) and 9 (N9) DAI with $M$. arenaria, during drought stress (Dro) and after UV exposure (UV), based on RNA-seq data

the phylogenetic analysis. These 16 AraEXLB8 genes from different Arachis genotypes showed 97.7\% of identity, with aligned segment sizes ranging from 740 to $755 \mathrm{bp}$. Overall, 19 nucleotide substitutions were detected, with the majority $(\mathrm{n}=12)$ occurring in exon 3 (Table 1; Supplementary Tables 6,7). Based on Blosum62 matrix, most 
Table 1 Haplotypes of AraEXLB8 gene in the 16 Arachis genotypes based on coding sequences segment (755 bp)

\begin{tabular}{|c|c|c|c|c|c|c|c|c|c|c|c|c|c|c|c|c|c|c|c|c|c|c|c|c|c|}
\hline \multicolumn{3}{|c|}{ SNP } & \multirow{2}{*}{$\begin{array}{l}1 \\
\text { E1 }\end{array}$} & \multirow[t]{2}{*}{2} & \multirow[t]{2}{*}{3} & \multirow[t]{2}{*}{4} & \multirow{2}{*}{$\begin{array}{l}5 \\
\text { E2 }\end{array}$} & \multirow{2}{*}{$\begin{array}{l}6 \\
\text { E3 }\end{array}$} & \multirow[t]{2}{*}{7} & \multirow[t]{2}{*}{8} & \multirow[t]{2}{*}{9} & \multirow[t]{2}{*}{10} & \multirow[t]{2}{*}{11} & \multirow[t]{2}{*}{12} & \multirow[t]{2}{*}{13} & \multirow[t]{2}{*}{14} & \multirow[t]{2}{*}{15} & \multirow[t]{2}{*}{16} & \multirow[t]{2}{*}{17} & \multirow{2}{*}{$\begin{array}{l}18 \\
\text { E4 }\end{array}$} & \multirow[t]{2}{*}{19} & \multicolumn{4}{|c|}{ Genome type } \\
\hline $\mathrm{H}$ & Fs & Fr & & & & & & & & & & & & & & & & & & & & A & B & K & $\mathrm{AB}$ \\
\hline H1 & 5 & 31 & $\mathrm{G}$ & C & G & C & C & A & $\mathrm{G}$ & A & C & A & A & C & A & A & $\mathrm{T}$ & C & C & C & C & - & - & - & 5 \\
\hline $\mathrm{H} 2$ & 1 & 6 & $*$ & $*$ & $*$ & $*$ & $*$ & $*$ & $*$ & $*$ & $*$ & $*$ & $*$ & $*$ & $*$ & $*$ & C & $*$ & $*$ & $*$ & * & 1 & - & - & - \\
\hline $\mathrm{H} 3$ & 1 & 6 & $*$ & $*$ & $\mathrm{~T}$ & $\mathrm{~T}$ & $*$ & * & $*$ & $*$ & $*$ & $*$ & $*$ & $*$ & $*$ & $*$ & C & * & * & $*$ & * & 1 & - & - & - \\
\hline H4 & 1 & 6 & $*$ & $\mathrm{~T}$ & $*$ & $*$ & $*$ & $*$ & $*$ & $*$ & $*$ & G & $*$ & $*$ & $*$ & G & $*$ & $*$ & $*$ & $*$ & $*$ & - & - & - & 1 \\
\hline H5 & 1 & 6 & $*$ & $*$ & $\mathrm{~T}$ & $*$ & $*$ & $\mathrm{~T}$ & $*$ & $*$ & $*$ & $*$ & C & $*$ & $*$ & $*$ & C & $\mathrm{G}$ & $*$ & G & $\mathrm{T}$ & - & - & 1 & - \\
\hline H6 & 3 & 19 & $*$ & $*$ & $\mathrm{~T}$ & $*$ & $*$ & $*$ & $\mathrm{~T}$ & $*$ & G & $*$ & $*$ & $*$ & $\mathrm{~T}$ & $*$ & C & $*$ & $\mathrm{~T}$ & $\mathrm{G}$ & $\mathrm{T}$ & 2 & 1 & - & - \\
\hline H7 & 1 & 6 & $*$ & $*$ & $\mathrm{~T}$ & $*$ & G & $\mathrm{C}$ & $*$ & $\mathrm{~T}$ & $*$ & $*$ & $\mathrm{C}$ & $\mathrm{T}$ & $*$ & $*$ & $\mathrm{C}$ & $*$ & $*$ & G & $\mathrm{T}$ & - & 1 & - & - \\
\hline H8 & 3 & 19 & A & $*$ & $\mathrm{~T}$ & $*$ & $*$ & $*$ & $*$ & $*$ & $*$ & $*$ & C & $\mathrm{T}$ & $*$ & $*$ & C & $*$ & $*$ & $\mathrm{G}$ & $\mathrm{T}$ & 1 & 2 & - & - \\
\hline
\end{tabular}

$H$ Haplotype; SNP single nucleotide polymorphism; $F s$ simple frequency; $F r$ relative frequency $(\%) ; E$ exon; * the same base of the first haplotype (H1)

of substitutions can be considered relatively conservative. Although four SNPs $(1 ; 2 ; 11$ and 13) were identified as non-synonymous/non-conservative (Supplementary Tables 6, 7), these nucleotide substitutions did not seem to change protein conformation, as suggested by the AraEXLB8 3D protein modeling predicted for four species (A. duranensis; A. stenosperma; A. ipaënsis and A. batizocoi) which showed no differences among their tertiary structure (data not shown). Interestingly, even without any putative effect on protein folding/structure, SNPs 11 and 13 occurred in the vicinity of a catalytic site presented in the crystal structure of a native Zea mays EXPB1 (Yennawar et al. 2006).

Eight haplotypes were further identified without any recombination event occurring (Table 1). Haplotype 1 (H1) stands out as it comprised all tetraploid genotypes (AB) analyzed here, with the highest SNP frequency being $31 \%$. Other haplotypes included only wild diploids, such as the H6 with two A (A. villosa and A. stenosperma) and one B (A. gregoryi) genome species, while the $\mathrm{H} 8$ comprised one A (A. cardenasii) and two B (both A. ipaënsis) species.

Phylogenetic analysis conducted with the above 16 AraEXLB8 coding sequences showed two distinct groups, with a posterior probability support value from 0.52 to 1, based on Bayesian Inference analysis (Supplementary Fig. 6). The first clade comprised all six tetraploids [AB: wild $(n=1)$, synthetic $(n=1)$, and cultivated $(n=4)$ ] derived from two distinct genotypes of A. duranensis (A genome) whilst the second clade was composed exclusively of diploids species with different genomes (A, B, and K).

\section{$A r a E X L B 8$ expression in response to stresses}

The expression levels of AraEXLB 8 analyzed by qRT-PCR in roots collected at the endpoint of the dry-down assay showed a significant and general upregulation in response to water deficit in all of the 13 genotypes studied (Fig. 4a).
Notably, the three wild species with higher expression $(A$. gregoryi, A. villosa and A. stenosperma with $\mathrm{RQ}=41.5-$; 16.3- and 11.5-fold, respectively) are those that were grouped as H6 (Table 1).

Regarding the response to RKN, the AraEXLB8 expression in roots during early stages of $M$. arenaria infection (3 DAI) displayed a clear contrasting behavior in the three Arachis species tested (Fig. 4b): upregulation (3.2-fold) in the RKN-resistant $A$. stenosperma and downregulation in the susceptible $A$. hypogaea 'Runner' (0.3-fold) and in the moderately resistant $A$. duranensis (0.6-fold). Despite the current lack of knowledge on the mechanisms through which AraEXLB8 might operate to contribute to RKN resistance, our results showed a clear trend between AraEXLB8 expression and plant resistance since the species displaying the lowest expression was the least resistant (A. hypogaea 'Runner') and the species with the highest expression was the most resistant (A. stenosperma). Distinct expression behavior was also observed in response to UV treatment in leaves, where AraEXLB8 was highly induced in A. stenosperma (24.3-fold) but to a lesser extent, in A. duranensis and A. hypogaea 'Runner' (5.3- and 1.8fold, respectively) (Fig. 4c).

To depict the spatial distribution of AraEXLB8 transcripts in roots, eight Arachis genotypes were analyzed by in situ hybridization (ISH) using samples collected at the endpoint of the dry-down or few days after $M$. arenaria inoculation. RNA was preserved in all samples as shown by the orange acridine treatment (Fig. 5a). Regardless of the morphological disorganization of the root cells due to drought stress, ISH signals were detected in the outer cortical and epidermal cells, with evident signals in A. gregoryi (Fig. 5b) and A. villosa (Fig. 5c), and lack of signals in well-watered control roots (Fig. 5d). In RKN-inoculated roots, AraEXLB8 transcripts were mostly present in the phloem and surrounding endodermal cells, with signals more evident in A. stenosperma (Fig. 5e) than in $A$. 
Fig. 4 Relative quantification (RQ) of AraEXLB8 transcripts. Expression profiles of AraEXLB8 determined by qRTPCR in a roots of 13 Arachis genotypes at the NTR around 0.3; in A. hypogaea 'Runner', $A$. duranensis and A. stenosperma b roots inoculated with $M$. arenaria at $3 \mathrm{DAI}$ and $\mathbf{c}$ leaves $24 \mathrm{~h}$ after UV treatment. Asterisks significantly regulated genes; letters statistical differences between genotype samples

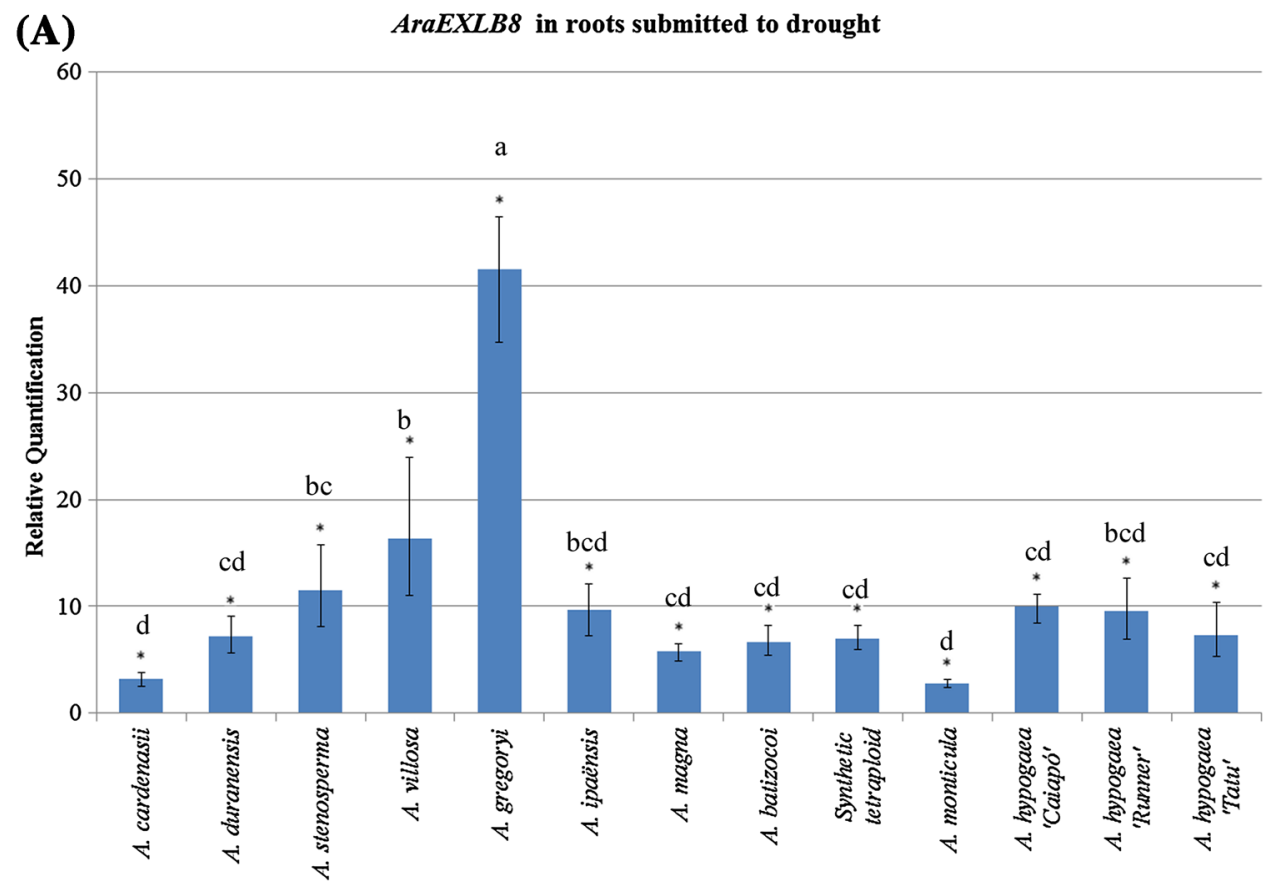

(B) $A r a E X L B 8$ in roots submitted to RKN-

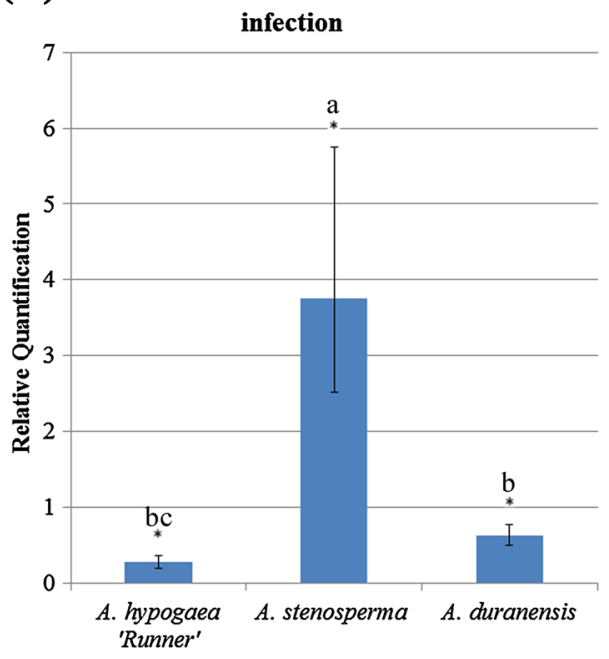

(C) $A r a E X L B$ in leaves submitted to UV stress

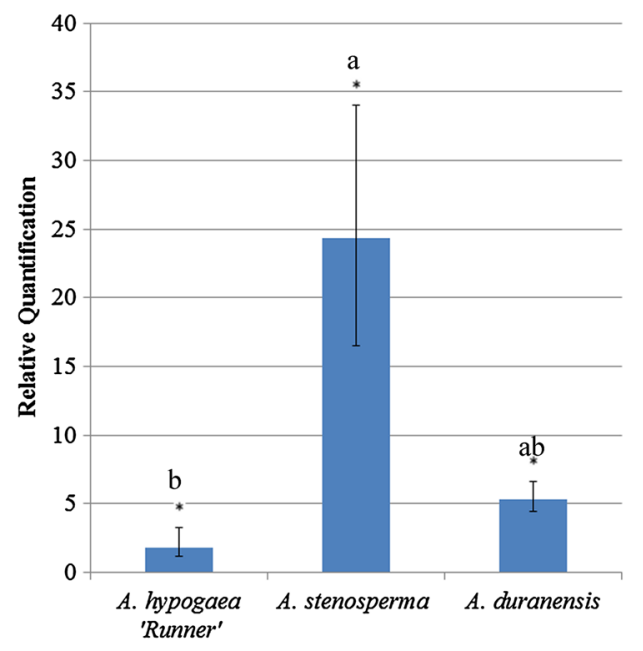

hypogaea 'Runner' (data not shown). No signals were observed in stressed samples hybridized with a sense (T7) probe (Fig. 5f). Hybridization signals showed different intensity depending on the plant genotype and tissue accordingly to the stress imposed.

\section{Overexpression of $A d E X L B 8$ in $G$. max composite plants}

Three weeks after Agrobacterium rhizogenes transformation, G. max composite plants developed transgenic hairy roots at the wounding sites (with an efficiency of 78 and $60 \%$ with empty and pPZP-AdEXLB8 vectors, respectively), which were inoculated with 1,000 M. javanica juveniles. After 60 days, the amount of eGFP-positive roots per composite plant varied between empty and pPZPAdEXLB8 vectors (36 and 19\% of the total hairy roots, respectively) (Supplementary Table 8).

Meloidogyne javanica juveniles were able to complete at least one full life cycle in G. max transgenic hairy roots, as all of the eGFP-positive roots transformed by the empty vector showed gall and egg mass formation, with an average of 19 galls per gram of root (Fig. 6a, b; Supplementary Table 8 ). Conversely, galls were poorly observed in the eGFP-positive roots transformed with pPZP-AdEXLB8 (average of 3 galls per gram of root), which constitutes a significant reduction of $82 \%$ in the number of galls compared to the empty vector control, with results varying for 

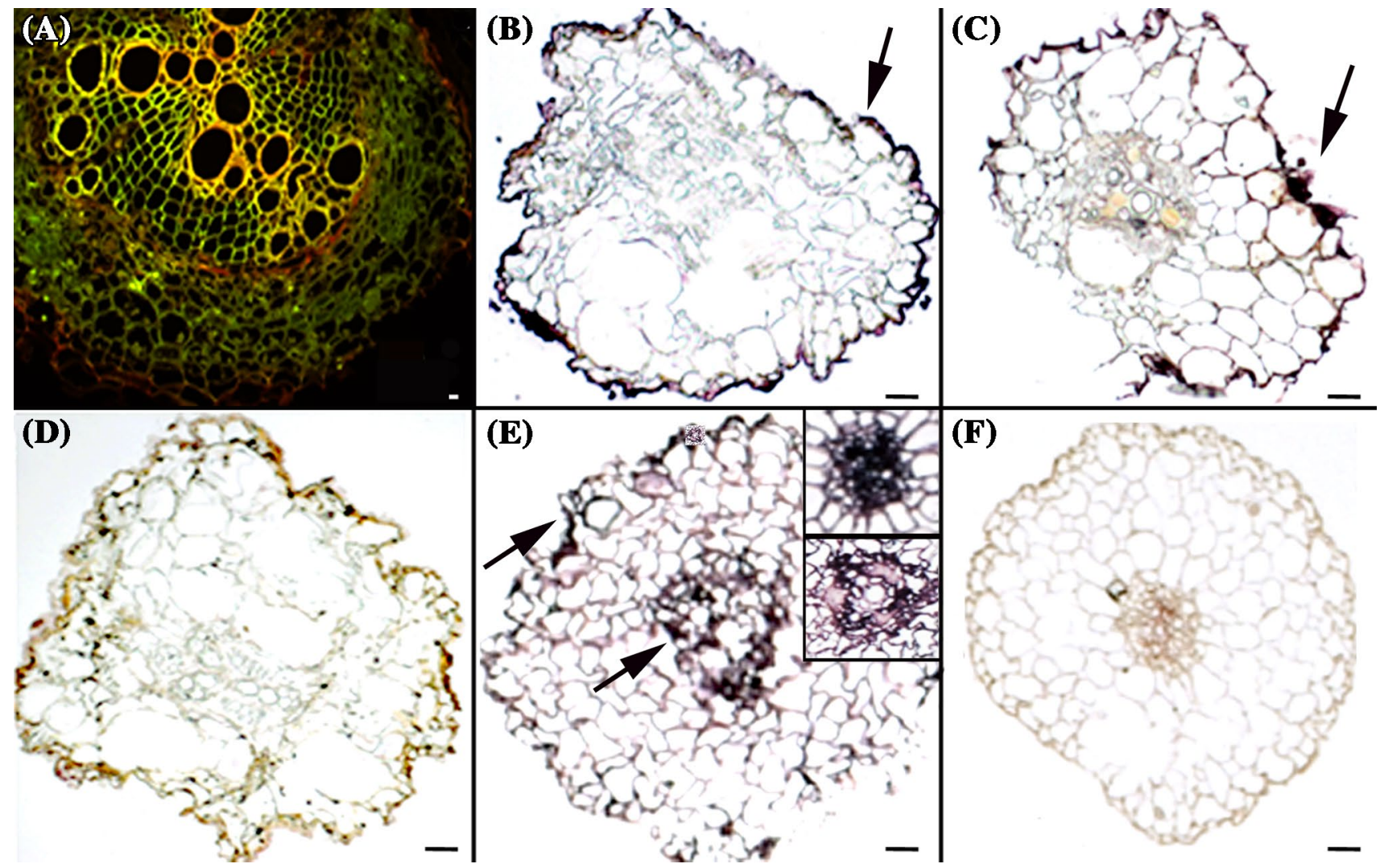

Fig. 5 AraEXLB8 in situ hybridization. a Preserved RNA (red) after acridine orange treatment in root cells of a non hybridized RKNinoculated A. stenosperma root (3 DAI). Drought-stressed roots of b $A$. gregoryi and $\mathbf{c} A$. villosa with hybridization signals detected in cortical and epidermal root cells. d Well-watered (control) roots of A. gregoryi, with no hybridization signals. e RKN-inoculated roots

different transformation events (Fig. 6c-e; Supplementary Table 8).

\section{Discussion}

Expansin induction as a preliminary step towards plant adaptation to environmental constraints and pathogen attack has been suggested as one of the mechanisms underlying plant resilience to overcome such stresses (Marowa et al. 2016). Despite their potential role in enhancing plant performance under adverse conditions, molecular and functional characterization of the expansin genes remains incomplete for most species. In this study, expansin superfamily organization, expression dynamics, and evolution was investigated in wild Arachis species known for harboring resistance to a large range of biotic and abiotic stresses (Bertioli et al. 2011). Their involvement in responses to drought, nematode infection, and UV treatment is further supported by current results. showing evident signals in the vascular cylinder and surrounding endodermal cells, as well as some signals in epidermal cells, of $A$. stenosperma (3 DAI). f $A$. stenosperma inoculated roots (3 DAI) with no signals after T7 hybridization with the sense (T7) probe (technical control). ISH signals indicated by arrows. Bars $=20 \mu \mathrm{m}$

\section{WGD contributed to the expansion of Arachis expansins}

The number of expansin genes herein identified in $A$. duranensis (40) and A. ipaënsis (44) was consistent with that previously described for other legumes which share a genome duplication with Arachis at the estimated time of Papilionoid origin (Shirasawa et al. 2013), as M. truncatula (42), P. vulgaris (36) and G. max (75). The higher number of expansin genes in G. max may reflect its later lineage-specific duplication. Arachis expansins could be further classified into the four subfamilies as proposed by (Kende et al. 2004). However, while EXPA, EXPB and EXLA formed highly supported monophyletic groups, the monophyly of EXLB was not highly supported and EXLBs were separated into two groups interspersed by EXLAs in the main phylogenetic analysis (Fig. 1a). Interestingly, a similar nonsupport of EXLB monophyly and a separation in two subgroups was also observed in a recent Bayesian phylogeny including Amborella, Arabidopsis and Oryza (Seader et al. 2016). However, this separation of EXLBs in two groups is only supported by low bootstrap (Fig. 1a) or 

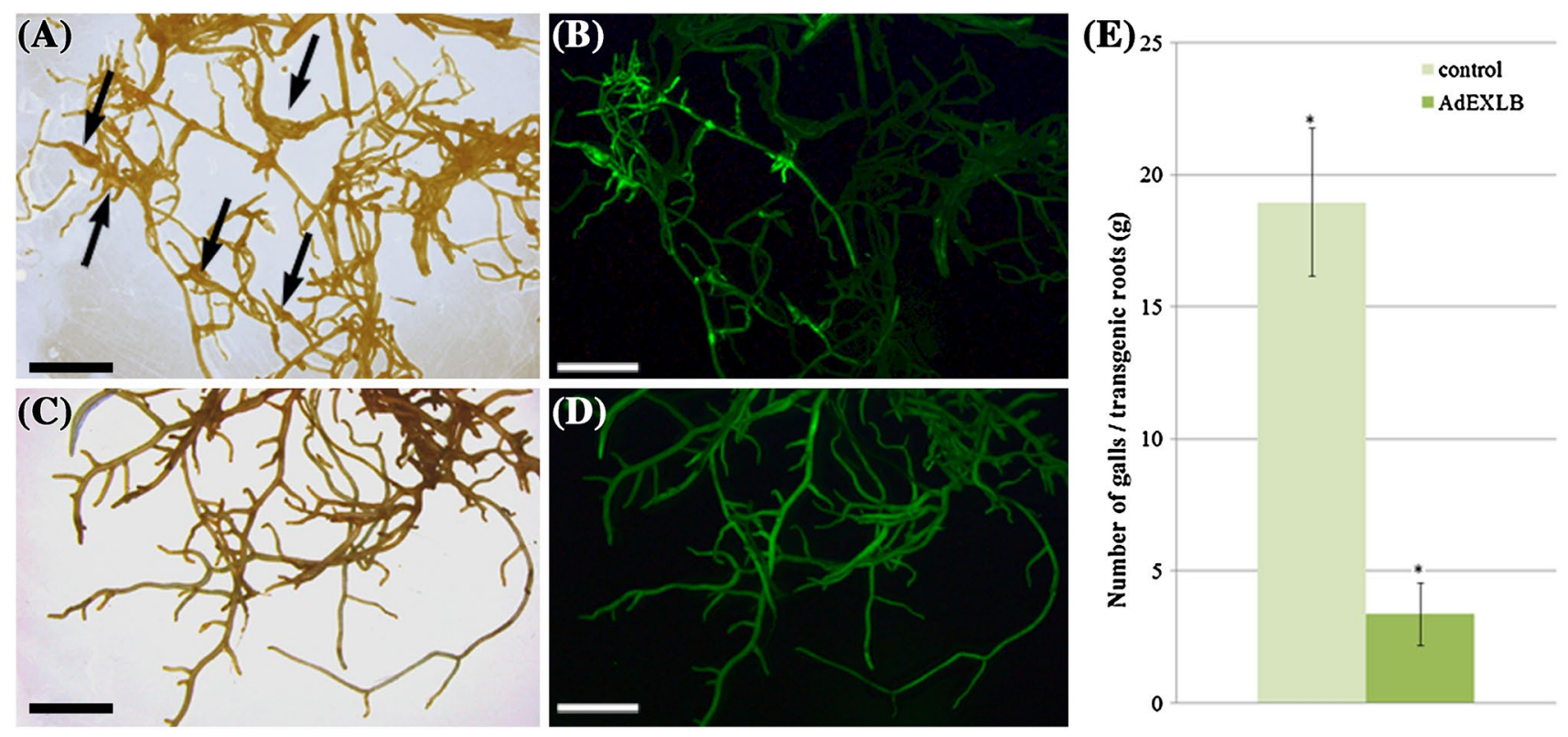

Fig. 6 Glycine max hairy roots infected with Meloidogyne javanica at 60 DAI. a, b Hairy roots transformed with empty and $\mathbf{c}, \mathbf{d}$ pPZPAdEXLB8 binary vectors under $\mathbf{a}$ and $\mathbf{c}$ white light and $\mathbf{b}$ and $\mathbf{d}$
$480 \pm 30 \mathrm{~nm}$ excitation GFP filter. e Number of galls per gram of hairy roots. Galls are indicated by arrows. Bars $=5 \mathrm{~mm}$

in expansin superfamily expansion (Sampedro et al. 2005; Zhu et al. 2014). Hence, present results support previous evidence that the expansion of the plant expansin superfamily is lineage-specific, and is mostly due to whole genome duplications in eudicots and tandem duplication in monocots (Sampedro et al. 2006). Retention and diversification of gene copies after duplications is one of the main forces in the evolution of higher plants (Freeling 2009; Rodgers-Melnick et al. 2012) and could be associated with subfunctionalization or neofunctionalization of expansin gene duplicates in angiosperms, resulting, for example, in differences in cell wall composition of grasses compared with eudicots (Cosgrove 2015; Sampedro et al. 2015).

In the Arachis expansin subfamily, it seems that the abundance of EXPA genes, distributed in every chromosome of both Arachis species, might be a result of multiple duplication events, with WGD being the major contributor for their abundance. Conversely, the majority of EXPB gene copies derived from tandem duplications. These duplications were probably responsible for the large EXPB cluster observed in chromosomes 2 of both species. In general, tandem duplications are expected to have a lower impact on the genome than WGD that initially doubles the number of all genes. The EXLB subfamily appears to have expanded specifically in legume species, ranging from 4 to 15 members per genome, whilst in other eudicots, they range from 1 to 4 (Supplementary Table 3). Moreover, in monocots, EXLB duplication showed a different evolution pattern, lacking in Zea mays (Zhang et al. was the predominant contributor with a major influence 
2014c) and having only one representative in $O$. sativa (Sampedro et al. 2005). Herein, the results showed that $50 \%$ of the EXLB genes were assigned to the WGD category of duplication. Actually, the EXLB that are mainly concentrated on chromosome 1 are part of a long block of duplicated genes conserved with chromosome 5. The remnant of this WGD are the couples of duplicates EXLB5 and EXLB7 on chromosome 1 that correspond to EXLB8 and EXLB6 on chromosome 5. This observation holds true for both Arachis species. The following series of events for the expansion of EXLBs in Arachis can thus be hypothesized: first an ancestral series of proximal or tandem duplications gave rise to an ancestral cluster of EXLBs. Then following a WGD event all these EXLBs were initially duplicated and distributed to the ancestors of chromosome 1 and 5 . Then, unbalanced gene loss led to preferential retention on chromosome 1 . This phenomenon of genome dominance after WGD leading to more genes retained on one of the duplicated regions has been frequently observed in plants (Murat et al. 2014). Overall, the higher abundance of EXLB genes in Arachis suggests a selective advantage to retain these multiple copies after a series of duplication. This implies that the EXLB genes could be part of wild Arachis mechanisms that ensure successful adaptability in their native adverse environments (Rodgers-Melnick et al. 2012). Consistent with the possible recruitment of EXLB for new functions, a strong positive selection was identified between AiEXLB5/AiEXLB8 gene copies, suggesting a functional divergence of this duplicated pair over the evolutionary history of (A) ipaënsis genome. The influence of positive selection in the expansin gene superfamily evolution has previously been reported for G. max, but not for other angiosperms such as (B) rapa or Z. mays (Krishnamurthy et al. 2014; Zhang et al. 2014c). This reinforces the present findings that AraEXLB5 and AraEXLB8 are stress-responsive genes (Brasileiro et al. 2015; Guimaraes et al. 2015), as positive selection is often observed in genes involved in defense/immunity and response to environmental constraints (Chen et al. 2010).

\section{Arachis expansin genes are widely modulated by stresses}

Genome-wide expression analysis demonstrated that expansin genes, regardless of the subfamily, were broadly expressed in roots of A. duranensis and A. stenosperma, and in leaves of $A$. stenosperma, and were widely responsive to external stimuli, thus confirming their involvement in stress responses. This expansin ubiquitous expression in roots and leaves was previously observed in many plant species, including legumes (Liu et al. 2015; Zhu et al. 2014), which is consistent with their role in root and root hair development, water and nutrient uptake (Che et al.
2016; Cho and Cosgrove 2004) and leaf growth and stomatal plasticity (Lü et al. 2013; Zhang et al. 2011). Moreover, the recently published $A$. hypogaea expression atlas based on 22 different tissue types and ontogenies (Clevenger et al. 2016) showed that 36 homologs out of the 38 A. duranensis expansin genes are expressed (absolute expression above 1) in at least one of the tissues that represent the full development (vegetative, reproductive and seed) of peanut. Some of these expansin genes also displayed preferential tissue expression (Clevenger et al. 2016): two AdEXPB genes were exclusively expressed in roots or in reproductive shoot tips, whilst five $A d E X L B$ transcripts were predominantly located in nodules. This indicates that, although expansins are implicated in a variety of plant processes, some Arachis genes might have evolved towards tissue-dependent functional specialization, as suggested for other species (Clevenger et al. 2016; Dal Santo et al. 2013; Lu et al. 2015; Zhang et al. 2014c). As the expression pattern of a gene is usually closely associated with its function, it is assumed that there are species-specific and/or stress-specific features in the biological functions of the Arachis expansin genes.

The involvement of expansins in adaptive responses to abiotic stresses has been widely reported and usually associated with the control of cell water loss and osmotic adjustment, probably by promoting cell wall extensibility, or with the general ROS-scavenging defense response to avoid oxidative damage (Marowa et al. 2016; Sasidharan et al. 2011). Here, the majority of the expansin genes were modulated according to the Arachis species and stress (drought and UV). Recently, Lu et al. (2015) also demonstrated that the responsiveness of Solanum lycopersicum expansins differs depending on the duration and type of stress imposed (drought, cold or heat). The combined evidence implies that different expansins could have distinct functions in response to abiotic stress via an ABA-independent pathway, as previously suggested (Cho and Cosgrove 2004).

Likewise, the overall expression levels of expansin transcripts upon a $M$. arenaria $\mathrm{RKN}$ infection revealed a general opposite profile in the RKN-resistant $A$. stenosperma and in the moderately RKN-resistant A. duranensis. This might reflect different mechanisms of resistance displayed by these species, which triggers the HR exclusively in $A$. stenosperma and an unknown defense response conferring partial resistance in A. duranensis (Proite et al. 2008). Further knowledge about the involvement of expansins in this parasitic association must, therefore, be pursued.

\section{AraEXLB8 is a promising molecular marker}

Among the A. duranensis expansin genes, AdEXLB8 exhibited a distinct and highly specific expression profile in response to both stresses, corroborating its previously 
association with Arachis drought tolerance (Brasileiro et al. 2015; Ding et al. 2014) and RKN resistance (Guimaraes et al. 2015; Tirumalaraju et al. 2011). AraEXLB8 nucleotide characterization evidenced the synonymous SNP15 as a promising molecular marker since it occurred exclusively on tetraploid genotypes (natural and synthetic). As previously suggested by Barkley et al. (2007), this type of polymorphism can be further exploited to design new molecular markers to distinguish cultivated tetraploids and wild diploids species in botanical and evolutionary studies. The haplotypes established here based on the AraEXLB8 nucleotide diversity indicated that they do not share ploidy level (diploidy and tetraploidy), plant life cycle (annual and perennial) or genome type (A, B, D and K), and do not necessarily reinforce phylogenetic proximities, as previously observed for A. magna and A. ipaënsis (Bechara et al. 2010; Krapovickas and Gregory 1994).

The AraEXLB8 gene conservation based on cDNA sequences was higher $(97.5 \%)$ than that observed in previous studies conducted with conserved regions or larger sampling of Arachis sequences (Bechara et al. 2010; Cunha et al. 2008; Moretzsohn et al. 2013), reinforcing the close phylogeny for the genotypes here observed. The high levels of conservation on AraEXLB8 cDNA sequences might reflect Arachis reproductive habits, such as autogamy and geocarpy that limit its crossability and seed dispersion (Bertioli et al. 2011; Krapovickas and Gregory 1994).

\section{$A r a E X L B 8$ is a promising candidate for plant genetic engineering}

The cDNA substitution characterizing the haplotype 6 (H6) can be further associated with the AraEXLB8 expression levels since the three H6 genotypes (A. stenosperma, A. villosa, and $A$. gregoryi) displayed the highest induction in response to drought. This overall induction of AraEXLB8 is coincident with the progressive decline in transpiration previously observed in wild Arachis species during a dry-down assay (Leal-Bertioli et al. 2012). Given that expansins are involved in root water uptake and growth (Lü et al. 2013; Zhang et al. 2011), it is possible that this protein, via rootto-leaf signaling, is implicated in both drought response mechanisms triggered upon the perception in roots of soil water availability restriction. Moreover, in situ detection of the AraEXLB8 transcripts indicates cell- and tissue-specific distribution, mostly in cortical and epidermal root cells, the primary site of water decrease perception. These cells might undergo morphology changes to avoid primary tissue damage by increasing expression of drought-responsive genes, such as AraEXLB8, involved in perception and signaling of this stress.

Several studies associate the EXPA, EXPB and EXLA genes with drought perception and adaptation (Marowa et al. 2016), but few reports have identified EXLB as drought-responsive genes. Previously, our group reported that $A r a E X L B 8$ is highly upregulated in response to water deficit in roots and leaves of A. duranensis and A. magna (Brasileiro et al. 2015) with its induction considered to be a preliminary step toward drought acclimation through adjusting cell wall extensibility (Harb et al. 2010). The responsiveness of AraEXLB8 orthologs to water deficit was also observed in roots of a drought-tolerant A. hypogaea genotype (Ding et al. 2014) and other legumes species, $M$. truncatula (Zhang et al. 2014a). Here, the increased expression levels of AraEXLB8 in the 13 Arachis genotypes studied, particularly in those belonging to the H6 group, associated with its specific distribution in surface cells of the roots, suggest that AraEXLB8 is involved in the network of molecular responses triggered by plants to circumvent dehydration damaging effects, making this gene a promising candidate for drought tolerance engineering.

Additional expression analysis in response to nematode infection in roots and UV treatment in leaves reinforce the prompt AraEXLB8 responsiveness to other stresses, with different levels of expression displayed by the Arachis genotypes that harbor different levels of pathogens resistance. The upregulation of AraEXLB8 orthologs in response to nematode infection was also reported in the Arachis RKNresistant genotypes (Guimaraes et al. 2015; Tirumalaraju et al. 2011) as well as the downregulation of a $P$. vulgaris $E X L B$ gene at the later stages of its compatible interaction with $M$. incognita (Santini et al. 2016). The shift in the spatial distribution of the AraEXLB8 transcripts in RKNinoculated Arachis roots, comparing to those detected in drought stressed, indicates the precise and stress-dependent regulation of this gene. The presence of AraEXLB8 in some cells of vascular bundles reinforces its involvement in RKN infection response, as nematodes in the early parasitic stages (J2) are mainly located within the differentiating vascular bundle (Ozalvo et al. 2014). These results suggest that, regardless of the mechanism of resistance displayed, the AraEXLB8 gene might have a more downstream role in the pathogen triggering response (PTI), and reinforces the need for a further understanding of molecular, biochemical and cellular events that culminate in pathogen resistance. The notable induction of AraEXLB8 in A. stenosperma leaves in response to UV can be also associated with the general ROS-scavenging and signal transduction pathways that triggered changes in the expression of some genes, as expansins, and in the production of secondary metabolites, as resveratrol, to control numerous biological process, including pathogen defense (Baxter et al. 2014; Han et al. 2015; Lopes et al. 2013), and therefore, contributing to the HR resistance mechanism of A. stenosperma, as previously suggested (Guimaraes et al. 2010; Morgante et al. 2013). UV irradiation and other physical stresses induce 
'cross-tolerance' responses that are commonly associated with pathogen defense and reactions to wounding, promoting increased resistance to pathogens (Mintoff et al. 2015).

In planta functional validation of $A d E X L B 8$ was carried out in G. max composite plants since A. hypogaea stable transformation is currently limited and time-consuming (Brasileiro et al. 2014; Kuma et al. 2015). This approach allowed the successful $A d E X L B 8$ overexpression in hairy roots, leading to a remarkable reduction in the number of galls produced by $M$. javanica. Like $M$. arenaria, the $M$. javanica RKN causes important damages in host plants, reducing drastically the productivity of many tropical crops, and it is particularly aggressive in $G$. $\max$, the most important commodity in the world (Beneventi et al. 2013). Since each hairy root obtained is an independent transformation event, a relative high number of eGFP-positive events could be analyzed, reinforcing that G. max composite plants are an efficient and fast system to validate Arachis RKN resistance candidates (Kuma et al. 2015). Moreover, the selection of transgenic events by eGFP fluorescence allowed the assessment of RKN infection uniquely in AdEXLB8-expressing roots, increasing the accuracy and speed of the analysis.

The literature is contradicting in terms of expansin roles in plant-microbe interactions, as the suppression of specific expansin genes reduced the infection by either pathogens, including nematodes (Ding et al. 2008; Gal et al. 2006; Marowa et al. 2016), or symbionts, as arbuscular mycorrhizal fungus (Dermatsev et al. 2010), whilst our results suggest that the overexpression of AraEXLB8 gene mediates RKN defense in G. $\max$ roots. The extent to which these non-enzymatic proteins act as physical barriers against microbes or as a source of signals to activate defense responses remains unclear, albeit their induction by auxin could be an underlying mechanism that should be considered (Sasidharan et al. 2011). The preferential expression of the Arachis EXLB genes in rhizobium-induced nodules (Clevenger et al. 2016) besides their overall induction upon RKN infection may indicated that the $E X L B$ genes are common components in the establishment of both parasitism and symbiosis and seem to play an important role in plant-microbe interactions. In fact, besides expansins, it has been demonstrated that rhizobia and RKN interactions with legumes share a significant number of target genes and display similar molecular mechanisms of symbiosis and pathogenesis to facilitate successful colonization (Damiani et al. 2012).

The effectiveness of $A d E X L B 8$ overexpression to reduce a RKN infection in stable transgenic plants of soybean and other legumes will enable the assessment of its potential to foster crop improvement for the mitigation of both, abiotic and biotic stresses, which is of utmost importance in the face of the predicted climate changes.

\section{Materials and methods}

\section{Identification of the Arachis expansin superfamily and phylogenetic analysis}

The protein sequences of A. thaliana and G. max expansins (http://www.personal.psu.edu/fsl/ExpCentral) were used to retrieve $A$. duranensis and $A$. ipaënsis expansin sequences from genome assemblies available in PeanutBase (http:// peanutbase.org/) Genome Threader software with default parameters (Gremme et al. 2005). Additional sequences were obtained from this database using "expansin" as keyword and TBLASTN searches (e-value 1e-3) against the genomes. The presence of the canonical expansin domains DPBB (PF03330) and CBM63 (PF01357) (Cosgrove 2015) was examined using the CDART tool (http://ncbi.nlm.nih. gov/Structure/lexington/lexington.cgi) and only sequences containing both of the domains were retained.

For phylogenetic analysis, all of the putative Arachis expansin protein sequences were aligned using MAFFT software, including sequences from $P$. vulgaris, $M$. truncatula and G. max (https://phytozome.jgi.doe.gov/pz/portal. html) and Arabidopsis thaliana (https://www.arabidopsis. org). To provide a more accurate alignment and construction of the phylogenetic tree, sequences with over $90 \%$ similarity were trimmed with trimAl (Capella-Gutiérrez et al. 2009). Phylogenic trees were constructed using the Maximum-Likelihood method as implemented in the RAxML software (Stamatakis 2006), with an automatic search of the fittest model and parameters as well as a bootstrap search automatically stopped upon congruence. The nomenclature of $A$. duranensis and A. ipaënsis expansins was given according to the synteny between the species and classification in subfamilies accordingly to Kende et al. (2004).

\section{Genomic distribution, duplication pattern, and structure of expansin genes}

The location of the putative expansin genes in A. duranensis and A. ipaënsis chromosomes was obtained from gene model annotations (http://peanutbase.org/) and all predicted protein sequences were submitted to self and all-against-all BLASTP analyses (e-value 1e-10). The expansins distribution and respective gene duplication patterns in chromosomes were analyzed by the MCScanX software with default parameters (Wang et al. 2012) and the results were formatted with custom perl script to Circos format (http:// circos.ca/) for graphical representation. To detect positive selection between genes, the ratio of $\mathrm{Ka} / \mathrm{Ks}$ between the gene copies of expansin sequences was computed using the Nei-Gojobori statistics (Nei and Gojobori 1986), as implemented in McScanX. 
Arachis expansin gene exon/intron organization retrieved from PeanutBase (http://peanutbase.org/) was submitted to GSDS software (http://gsds.cbi.pku.edu.cn/). InterPro and SignalP were used for protein domain and signal peptide prediction, respectively, and the isoelectric points and molecular weights of the proteins obtained from ExPASy (http://expasy.org/).

\section{RNA-seq expression profiling of Arachis expansin genes}

Illumina RNA-seq data previously obtained by our group from roots of A. duranensis and A. stenosperma plants submitted to drought (available at http://peanutbase.org) or inoculated with RKN M. arenaria (collected at 3, 6 or 9 days after inoculation; DAI) (Guimaraes et al. 2015) or from leaves of A. stenosperma submitted to UV exposure (unpublished results) were used for the expansin genes expression analysis. The genome of A. duranensis was used to create an index with the Kallisto program (version 0.42.3) and only high quality reads from the transcriptome were then used for the quantification against the created index, with default settings (Bray et al. 2016). For the differential expression analysis, the edgeR package (Robinson et al. 2010) was employed. The $\log 2$ of fold-change value between control and treated samples in both species was used for the heatmap generation using heatmap2R package (Warnes et al. 2015).

\section{AraEXLB8 diversity in Arachis genotypes}

Total RNA was isolated from young leaves of 13 Arachis genotypes (Supplementary Table 9) and the corresponding cDNAs used for RT-PCR as described by Morgante et al. (2011), employing AraEXLB8-flanking primers (EXPF 5'-AGTGCTTCATCTCAAAATGGAACT-3' and EXPR 5'-TCTATTCAAGCTGGATTTGAGTATCA-3'). Sequenced amplification products were aligned using the Sequencher software (Gene Codes, Ann Arbor, USA) with three additional available AraEXLB8 sequences from $A$. duranensis and A. ipaënsis (http://peanutbase.org/gbrowse) and A. hypogaea 'Tifrunner' [GO326087.1; (http://www. ncbi.nlm.nih.gov)]. Nucleotide substitutions were defined by BLOSUM62 matrix (Henikoff and Henikoff 1992) and hydrophobicity (Kyte and Doolittle 1982) while DNA sequence variation by DnaSP software (http://www.ub.es/ dnasp/).

Phylogenetic analysis was conducted using the above 16 AraEXLB8 coding sequences and four additional outgroups (G. $\max$ GI:955303815; P. vulgaris GI:593787101; C. arietinum GI:828330493 and M. truncatula Medtr5g013440.1). The phylogeny was constructed by Bayesian Inference using MrBayes program (http:// mrbayes.sourceforge.net/) with default parameters and performed with 20 million generations. The phylogenetic model $(\mathrm{GTR}+\mathrm{I})$ was selected as the fittest model according to ModelTest and the consensus trees were inspected in FigTree software (http://tree.bio.ed.ac.uk/software/figtree/).

\section{AraEXLB8 qRT-PCR analysis and in situ transcripts detection}

Dry-down assay

Normalized transpiration rates (NTR) were determined for 4-week-old plants of 13 Arachis genotypes (Supplementary Table 9) throughout a soil gradual water deficit assay (drydown), as previously described (Leal-Bertioli et al. 2012). Roots were collected when each drought stressed (DS) individual reached a NTR value around 0.3 relative to wellwatered (WW) controls. Samples from three individuals were assembled forming three independent biological replicates for each treatment (WW and DS), and per genotype.

\section{RKN inoculation assay}

Arachis stenosperma, A. duranensis and A. hypogaea 'Runner' 4-week-old plants (Supplementary Table 9) were inoculated with $M$. arenaria race 1 at juvenile stage 2 (J2) as described by Morgante et al. (2013). Root samples from five individuals were collected at zero (control), 3 DAI (stressed), and assembled to form two independent biological replicates per treatment and genotype.

\section{UV assay}

Young leaves were collected from 7-week-old plants of A. stenosperma, A. duranensis and A. hypogaea 'Runner' (Supplementary Table 9) and submitted to UV-C light treatment for $2 \mathrm{~h}$ and $30 \mathrm{~min}$, essentially as described by Lopes et al. (2013). Samples from five individuals were collected before (control) and $24 \mathrm{~h}$ after (stressed) UV treatment and assembled to form three independent biological replicates for each treatment and genotype.

\section{Quantitative RT-PCR (qRT-PCR) analysis}

Total RNA was extracted from samples and the cDNA synthesized as previously described (Morgante et al. 2011). The qRT-PCR reactions were conducted in a 7300 RealTime PCR System (Applied Biosystem) using primers of the A. duranensis ELXB8 (AdEXLB8) gene, as previously described (Brasileiro et al. 2015). In accordance to Morgante et al. (2011), ACT1 and UBI2 were used as reference genes for samples subjected to dry-down whereas $60 \mathrm{~S}$ and GAPDH to RKN inoculation and UV treatments. The relative quantification (RQ) of transcripts was determined as 
described by Brasileiro et al. (2015) and statistically tested using one-way ANOVA followed by Tukey's test.

\section{In situ hybridization (ISH) analysis}

Root fragments were isolated and processed in accordance to (Morgante et al. 2013). A 659 bp cDNA sequence of AdEXLB8 was amplified (EXPuniF: 5'-ACTGCCAGTCAC TTGGAACC-3' and Exp464R: 5'-AGATCCATTCCGCCA TAGC-3'), cloned into pGEM ${ }^{\circledR}$-T Easy (Promega, Madison, USA) and used to produce dig-labeled RNA probes (DIG RNA Labeling Kit SP6/T7, Roche). Slides were then hybridized with $2 \mathrm{ng}$ of probe and hybridization sites were immunocytochemically detected as described (Morgante et al. 2013).

\section{Meloidogyne javanica bioassay in G. max composite plants overexpressing $A d E X L B 8$ gene}

The coding region (753 bp) of AdEXLB8 was cloned (Epoch Life Science, Texas, USA) under the control of the A. thaliana actin 2 promoter, at the XhoI restriction site of the binary vector pPZP-201BK-EGFP (Chu et al. 2014) containing an enhanced Green Fluorescent Protein (eGFP) to form the pPZP-AdEXLB8 vector. Agrobacterium rhizogenes strain 'K599', harboring pPZP-201BK-EGFP (empty) or pPZP-AdEXLB8, vectors was then used to generate G. $\max$ 'Williams 82 ' composite plants employing fresh bacterial paste inoculum (Chu et al. 2014), basically as described before (Kuma et al. 2015). Transgenic roots were identified one week later through eGFP fluorescence under GFP1 filter set on a M205 stereomicroscope (Leica Microsystems, Wetzlar, Germany) and the eGFP-negative roots were excised before plant transfer to a 3:1 sand:plant gel mixture (v:v) and acclimation in greenhouse conditions. Three-week-old composite plants were challenged with approximately $1000 \mathrm{~J} 2$ of $M$. javanica and the number of galls determined at 60 DAI. The qRT-PCR analysis of AdEXLB8 expression in eGFP-positive roots was conducted as described above. Outliers in galls counting data were removed by Grubbs' test and means compared by unpaired t-test using Graphpad software (http://graphpad. com/quickcalcs/ttest1/).

Acknowledgements We would like to thank Drs. Marília Santos, Márcio Moretzsohn, Roberto Togawa, Marcos Mota, and Regina Carneiro (Embrapa Cenargen; Brazil) and MSc. Paula Vasconcelos (UNESP; Brazil) for helpful contribution on bioassays and data analysis and Peggy Ozias-Akins (University of Georgia, USA) for providing pPZP-201BK-EGFP vector. The authors gratefully acknowledge support from 'Empresa Brasileira de Pesquisa Agropecuária' (Embrapa); 'Conselho Nacional de Desenvolvimento Científico e Tecnológico' (CNPq); and 'Fundação de Apoio à Pesquisa do DF' (FAPDF). EGJD is a recipient of a CNPq/PVE (Special Guest Researcher) grant from the Brazilian program 'Science without Borders'; LAG is a recipient of fellowships from CNPq and APZM, BMP and RBS from 'Coordenação de Aperfeiçoamento de Pessoal de Nível Superior' (CAPES).

Author Contributions Conceived and designed the experiments: LAG, ACGA, PMG and ACMB; Performed the experiments: BMP, MAPS and RBS; Analyzed the data: LAG, APZM, ACGA, LFAF and ACMB; Wrote the paper: LAG, APZM, ACGA, LFAF, EGJD, PMG and ACMB; Contributed reagents/materials/analysis tools: ACGA, EGJD, PMG and ACMB; Critically revised the manuscript: All authors.

\section{Compliance with ethical standards}

Conflict of interest The authors have declared that no competing interests exist.

Open Access This article is distributed under the terms of the Creative Commons Attribution 4.0 International License (http:// creativecommons.org/licenses/by/4.0/), which permits unrestricted use, distribution, and reproduction in any medium, provided you give appropriate credit to the original author(s) and the source, provide a link to the Creative Commons license, and indicate if changes were made.

\section{References}

Barkley NA, Dean RE, Pittman RN, Wang ML, Holbrook CC, Pederson GA (2007) Genetic diversity of cultivated and wild-type peanuts evaluated with M13-tailed SSR markers and sequencing. Genet Res 89:93-106. doi:10.1017/S0016672307008695

Baxter A, Mittler R, Suzuki N (2014) ROS as key players in plant stress signalling. J Exp Bot 65:1229-1240

Bechara MD et al (2010) Phylogenetic relationships in genus Arachis based on ITS and 5.8 S rDNA sequences. BMC Plant Biol 10:255-255

Beneventi M et al (2013) Transcription profile of soybean-root-knot nematode interaction reveals a key role of phythormones in the resistance reaction. BMC Genomics 14:322

Bertioli DJ, Seijo G, Freitas FO, Valls JFM, Leal-Bertioli SCM, Moretzsohn MC (2011) An overview of peanut and its wild relatives. Plant Genet Resour 9:134-149 doi:10.1017/ S1479262110000444

Bertioli DJ et al. (2016) The genome sequences of Arachis duranensis and Arachis ipaensis, the diploid ancestors of cultivated peanut. Nat Genet 48:438

Brasileiro ACM, Araujo ACG, Leal-Bertioli SC, Guimaraes PM (2014) Genomics and genetic transformation in Arachis. Int J Plant Biol Res 2:1017

Brasileiro ACM et al. (2015) Transcriptome profiling of wild Arachis from water-limited environments uncovers drought tolerance candidate genes. Plant Mol Biol Rep. doi:10.1007/ s11105-015-0882-x

Bray NL, Pimentel H, Melsted P, Pachter L (2016) Near-optimal probabilistic RNA-seq quantification. Nat Biotechnol 34:525-527

Capella-Gutiérrez S, Silla-Martínez JM, Gabaldón T (2009) trimAl: a tool for automated alignment trimming in large-scale phylogenetic analyses. Bioinformatics 25:1972-1973

Che J, Yamaji N, Shen RF, Ma JF (2016) An Al-inducible expansin gene, OsEXPA10 is involved in root cell elongation of rice. Plant J 88:132. doi:10.1111/tpj.13237

Chen Q, Han Z, Jiang H, Tian D, Yang S (2010) Strong positive selection drives rapid diversification of R-genes in Arabidopsis relatives. J Mol Evol 70:137-148 
Chen X et al (2016) Draft genome of the peanut A-genome progenitor (Arachis duranensis) provides insights into geocarpy, oil biosynthesis, and allergens. Proc Natl Acad Sci USA. doi:10.1073/pnas.1600899113

Cho HT, Cosgrove DJ (2004) Expansins as agents in hormone action. In: Davies PJ (ed) Plant hormones. 3rd edn. Kluwer Academic, Dordrecht, pp 262-281

Chu Y, Guimaraes L, Wu C, Timper P, Holbrook C, Ozias-Akins P (2014) A technique to study Meloidogyne arenaria resistance in Agrobacterium rhizogenes -transformed peanut. Plant Dis 98:1292-1299

Clevenger JPC, Chu Y, Scheffler B, Ozias-Akins P (2016) A developmental transcriptome map for allotetraploid Arachis hypogaea. Front Plant Sci 7:1446

Cosgrove DJ (2015) Plant expansins: diversity and interactions with plant cell walls. Curr Opin Plant Biol 25:162-172

Cosgrove DJ (2016) Catalysts of plant cell wall loosening. F1000Research 5:F1000 Faculty Rev-1119. doi:10.12688/ f1000research.7180.1

Cunha FB, Nobile PM, Hoshino AA, Moretzsohn MdC, Lopes CR, Gimenes MA (2008) Genetic relationships among Arachis hypogaea $\mathrm{L}$. (AABB) diploid Arachis species with AA BB genomes. Genet Resour Crop Evol 55:15-20. doi:10.1007/ s10722-007-9209-6

Dal Santo S, Vannozzi A, Tornielli GB, Fasoli M, Venturini L, Pezzotti M, Zenoni S (2013) Genome-wide analysis of the expansin gene superfamily reveals grapevine-specific structural and functional characteristics. PLoS ONE 8:e62206

Damiani I et al (2012) Plant genes involved in harbouring symbiotic rhizobia or pathogenic nematodes. New Phytol 194:511-522

Dermatsev V et al (2010) Microarray analysis and functional tests suggest the involvement of expansins in the early stages of symbiosis of the arbuscular mycorrhizal fungus Glomus intraradices on tomato (Solanum lycopersicum). Mol Plant Pathol 11:121-135

Ding X, Cao Y, Huang L, Zhao J, Xu C, Li X, Wang S (2008) Activation of the indole-3-acetic acid-amido synthetase GH3-8 suppresses expansin expression and promotes salicylate-and jasmonate-independent basal immunity in rice. Plant Cell 20:228-240

Ding H, Zhang ZM, Qin FF, Dai LX, Li CJ, Ci DW, Song WW (2014) Isolation and characterization of drought-responsive genes from peanut roots by suppression subtractive hybridization.Electron J Biotechnol 17:304-310

Ding A, Marowa P, Kong Y (2016) Genome-wide identification of the expansin gene family in tobacco (Nicotiana tabacum). Mol Genet Genomics 291:1891-1907

Freeling M (2009) Bias in plant gene content following different sorts of duplication: tandem, whole-genome, segmental, or by transposition. Annu Rev Plant Biol 60:433-453. doi:10.1146/ annurev.arplant.043008.092122

Gal T, Aussenberg E, Burdman S, Kapulnik Y, Koltai H (2006) Expression of a plant expansin is involved in the establishment of root knot nematode parasitism in tomato. Planta 224:155162. doi:10.1007/s00425-005-0204-x

Gremme G, Brendel V, Sparks ME, Kurtz S (2005) Engineering a software tool for gene structure prediction in higher organisms. Inf Softw Technol 47:965-978

Guimaraes PM et al. (2010) A study of gene expression in the nematode resistant wild peanut relative, Arachis stenosperma, in response to challenge with Meloidogyne arenaria. Trop Plant Biol. doi:10.1007/s12042-010-9056-z

Guimaraes PM et al (2012) Global transcriptome analysis of two wild relatives of peanut under drought and fungi infection. BMC Genomics 13:387
Guimaraes PM et al (2015) Root transcriptome analysis of wild peanut reveals candidate genes for nematode resistance. PLoS ONE 10:e0140937

Han Y, Chen Y, Yin S, Zhang M, Wang W (2015) Over-expression of TaEXPB23, a wheat expansin gene, improves oxidative stress tolerance in transgenic tobacco plants. J Plant Physiol 173:62-71

Harb A, Krishnan A, Ambavaram MM, Pereira A (2010) Molecular and physiological analysis of drought stress in Arabidopsis reveals early responses leading to acclimation in plant growth. Plant Physiol 154:1254-1271

Henikoff S, Henikoff JG (1992) Amino acid substitution matrices from protein blocks. Proc Natl Acad Sci USA 89:10915-10919

Janila $\mathrm{P}$ et al. (2016) Genomic tools in groundnut breeding program: status and perspectives Front Plant Sci 7:289

Kende $\mathrm{H}$ et al (2004) Nomenclature for members of the expansin superfamily of genes and proteins. Plant Mol Biol 55:311-314

Krapovickas A, Gregory W (1994) Taxonomia del genero Arachis (Leguminosae). Bonplandia 8:1-186

Krishnamurthy P, Hong JK, Kim JA, Jeong M-J, Lee Y-H, Lee SI (2014) Genome-wide analysis of the expansin gene superfamily reveals Brassica rapa -specific evolutionary dynamics upon whole genome triplication. Mol Genet Genomics 290:521-530

Kuma KM et al (2015) A high efficient protocol for soybean root transformation by Agrobacterium rhizogenes and most stable reference genes for RT-qPCR analysis. Plant Cell Rep 34:1987-2000. doi:10.1007/s00299-015-1845-2

Kyte J, Doolittle RF (1982) A simple method for displaying the hydropathic character of a protein. J Mol Biol 157:105-132

Leal-Bertioli SCM et al (2012) The effect of tetraploidization of wild Arachis on leaf morphology and other drought-related traits. Environ Exp Bot 84:17-24

Liu Y, Zhang J, Li W, Guo C, Shu Y (2015) In silico identification, phylogeny and expression analysis of expansin superfamily in Medicago truncatula. Biotechnol Biotechnol Equip 30:1-7

Lopes RM, Silveira D, Gimenes MA, Vasconcelos PAS, Alves RBN, Silva JP, Agostini-Costa TS (2013) Characterization of resveratrol content in ten wild species of section Arachis, genus Arachis. Genet Resour Crop Evol 60:2219-2226

Lu Y, Liu L, Wang X, Han Z, Ouyang B, Zhang J, Li H (2015) Genome-wide identification and expression analysis of the expansin gene family in tomato. Mol Genet Genomics. doi:10.1007/s00438-015-1133-4

Lü P, Kang M, Jiang X, Dai F, Gao J, Zhang C (2013) RhEXPA4, a rose expansin gene, modulates leaf growth and confers drought and salt tolerance to Arabidopsis. Planta. doi:10.1007/ s00425-013-1867-3

Marowa P, Ding A, Kong Y (2016) Expansins: roles in plant growth and potential applications in crop improvement. Plant Cell Rep. doi:10.1007/s00299-016-1948-4

McQueen-Mason S, Durachko DM, Cosgrove DJ (1992) Two endogenous proteins that induce cell wall extension in plants. Plant Cell 4:1425-1433. doi:10.1105/tpc.4.11.1425

Mintoff S, Rookes J, Cahill D (2015) Sub-lethal UV-C radiation induces callose, hydrogen peroxide and defence-related gene expression in Arabidopsis thaliana. Plant Biol 17:703-711

Moretzsohn MC, Gouvea EG, Inglis PW, Leal-Bertioli SCM, Valls JFM, Bertioli DJ (2013) A study of the relationships of cultivated peanut (Arachis hypogaea) and its most closely related wild species using intron sequences and microsatellite markers. Ann Bot 111:113-126. doi:10.1093/aob/mcs237

Morgante C, Guimaraes P, Martins A, Araujo A, Leal-Bertioli S, Bertioli D, Brasileiro A (2011) Reference genes for quantitative reverse transcription-polymerase chain reaction expression studies in wild and cultivated peanut. BMC Res Notes 4:339 
Morgante CV et al (2013) A survey of genes involved in Arachis stenosperma resistance to Meloidogyne arenaria race 1. Funct Plant Biol 40:1298-1309

Murat F et al. (2014) Shared subgenome dominance following polyploidization explains grass genome evolutionary plasticity from a seven protochromosome ancestor with $16 \mathrm{~K}$ protogenes. Genome Biol Evol 6:12-33

Nei M, Gojobori T (1986) Simple methods for estimating the numbers of synonymous and nonsynonymous nucleotide substitutions. Mol Biol Evol 3:418-426

Ozalvo R et al (2014) Two closely related members of Arabidopsis 13-lipoxygenases (13-LOXs), LOX3 and LOX4, reveal distinct functions in response to plant-parasitic nematode infection. Mol Plant Pathol 15:319-332

Proite K, Carneiro R, Falcao R, Gomes A, Leal-Bertioli S, Guimaraes P, Bertioli D (2008) Post-infection development and histopathology of Meloidogyne arenaria race 1 on Arachis spp. Plant Pathol 57:974-980. doi:10.1111/j.1365-3059.2008.01861.x

Robinson MD, McCarthy DJ, Smyth GK (2010) edgeR: a Bioconductor package for differential expression analysis of digital gene expression data. Bioinformatics 26:139-140

Rodgers-Melnick E et al (2012) Contrasting patterns of evolution following whole genome versus tandem duplication events in Populus. Genome Res 22:95-105. doi:10.1101/gr.125146.111

Sampedro J, Cosgrove DJ (2005) The expansin superfamily. Genome Biol 6:242

Sampedro J, Lee Y, Carey RE, DePamphilis C, Cosgrove DJ (2005) Use of genomic history to improve phylogeny and understanding of births and deaths in a gene family. Plant J 44:409-419. doi:10.1111/j.1365-313X.2005.02540.x

Sampedro J, Carey RE, Cosgrove DJ (2006) Genome histories clarify evolution of the expansin superfamily: new insights from the poplar genome and pine ESTs. J Plant Res 119:11-21

Sampedro J, Guttman M, Li L-C, Cosgrove DJ (2015) Evolutionary divergence of $\beta$-expansin structure and function in grasses parallels emergence of distinctive primary cell wall traits. Plant $\mathrm{J}$ 81:108-120. doi:10.1111/tpj.12715

Santini L, Munhoz CdF, Bonfim MF Jr, Brandão MM, Inomoto MM, Vieira MLC (2016) Host transcriptional profiling at early and later stages of the compatible interaction between Phaseolus vulgaris and Meloidogyne incognita. Phytopathology 106:282-294

Sasidharan R, Voesenek LA, Pierik R (2011) Cell wall modifying proteins mediate plant acclimatization to biotic and abiotic stresses. Crit Rev Plant Sci 30:548-562
Seader VH, Thornsberry JM, Carey RE (2016) Utility of the Amborella trichopoda expansin superfamily in elucidating the history of angiosperm expansins. J Plant Res 129:199-207

Shirasawa K et al (2013) Integrated consensus map of cultivated peanut and wild relatives reveals structures of the $\mathrm{A}$ and $\mathrm{B}$ genomes of arachis and divergence of the legume genomes. DNA Res 20:173-184. doi:10.1093/dnares/dss042

Stamatakis A (2006) RAxML-VI-HPC: maximum likelihood-based phylogenetic analyses with thousands of taxa and mixed models. Bioinformatics 22:2688-2690

Tirumalaraju SV, Jain M, Gallo M (2011) Differential gene expression in roots of nematode-resistant and -susceptible peanut (Arachis hypogaea) cultivars in response to early stages of peanut rootknot nematode (Meloidogyne arenaria) parasitization. J Plant Physiol 168:481-492

Wang Y et al (2012) MCScanX: a toolkit for detection and evolutionary analysis of gene synteny and collinearity. Nucleic Acids Res 40:e49-e49

Warnes G, Bolker B, Bonebakker L, Gentleman R, Liaw W, Lumley T (2015) gplots: Various R Programming Tools for Plotting Data. R package version 2.16. 0. 2015.

Yennawar NH, Li L-C, Dudzinski DM, Tabuchi A, Cosgrove DJ (2006) Crystal structure and activities of EXPB1 (Zea m 1), a $\beta$-expansin and group-1 pollen allergen from maize. Proc Natl Acad Sci USA 103:14664-14671

Zhang X-Q, Wei P-C, Xiong Y-M, Yang Y, Chen J, Wang X-C (2011) Overexpression of the Arabidopsis $\alpha$-expansin gene AtEXPA1 accelerates stomatal opening by decreasing the volumetric elastic modulus. Plant Cell Rep 30:27-36

Zhang J-Y et al (2014a) Global reprogramming of transcription and metabolism in Medicago truncatula during progressive drought and after rewatering. Plant Cell Environ 37:2553-2576

Zhang S, Xu R, Gao Z, Chen C, Jiang Z, Shu H (2014b) A genomewide analysis of the expansin genes in Malus $\times$ Domestica. Mol Genet Genomics 289:225-236

Zhang W et al (2014c) Genome-wide identification and characterization of maize expansin genes expressed in endosperm. Mol Genet Genomics 289:1061-1074

Zhu Y, Wu N, Song W, Yin G, Qin Y, Yan Y, Hu Y (2014) Soybean (Glycine max) expansin gene superfamily origins: segmental and tandem duplication events followed by divergent selection among subfamilies. BMC Plant Biol 14:93 